\title{
INSTANTONS AND THE POTENTIAL OF THE TRIPLE-WELL SHAPE
}

\author{
K. RAJCHEL \\ Department of Physics of the High Pedagogical School, \\ Podchorązych 2, 30-084 Kraków, Poland \\ (Received April 12, 1990)
}

\begin{abstract}
Applications of instantons to compute quantum-mechanical problems with spinless particles are shown. Effectivness and correctness of this method as a non-perturbative method is compared with the variational method applied to the potential of the triple-well shape. Limits of applicability of instanton solutions to compute energy of the ground state are also considered.
\end{abstract}

PACS numbers: 03.65.-w

\section{Introduction}

In this section we deal exlusively with the theory of spinless particle of unit mass moving in one dimension. We start with the Lagrangian:

$$
L=\frac{1}{2}\left(\frac{\mathrm{d} x}{\mathrm{~d} t}\right)^{2}-V(x)
$$

and we choose the following boundary conditions:

$$
x\left(-\frac{t_{0}}{2}\right)=x_{i}, \quad x\left(\frac{t_{0}}{2}\right)=x_{f} .
$$

Our fundamental tool will be Feynman's sum over path (with $h=1$ ):

$$
\left\langle x_{f}\left|\mathrm{e}^{-\mathrm{i} H t_{0}}\right| x_{i}\right\rangle=N \int[\mathrm{D} x] \mathrm{e}^{\mathrm{i} S[x(t)]},
$$

where: $\left|x_{i}\right\rangle$ and $\left|x_{f}\right\rangle$ are position eigenstates, $H$ is the Hamiltonian, $t_{0}$ is a positive number, and $N$ is the normalization constant. 
If we expand the left-hand side of (1.2) in a complete set of energy eigenstates,

$$
H|n\rangle=E_{n}|n\rangle,
$$

then

$$
\left\langle x_{f}\left|\mathrm{e}^{-\mathrm{i} H t_{0}}\right| x_{i}\right\rangle=\sum_{n} \mathrm{e}^{-\mathrm{i} E_{n} t_{0}}\left\langle x_{f} \mid n\right\rangle\left\langle n \mid x_{i}\right\rangle
$$

We can separate the leading term in this expression for large $t_{0}$, if we change $t \rightarrow-\mathrm{i} \tau$. Then on the right-hand side of (1.2) we get:

$$
\mathrm{i} S[x(\tau)]=\int_{-\tau_{0} / 2}^{\tau_{0} / 2}\left[-\frac{1}{2}\left(\frac{\mathrm{d} x}{\mathrm{~d} \tau}\right)^{2}-V(x)\right] \mathrm{d} \tau
$$

and the so-called "Euclidean action" is equal to:

$$
S=\int_{-\tau_{0} / 2}^{\tau_{0} / 2}\left[\frac{1}{2}\left(\frac{\mathrm{d} x}{\mathrm{~d} \tau}\right)^{2}+V(x)\right] \mathrm{d} \tau
$$

Thus the final form of (1.2) is

$$
\left\langle x_{f}\left|\mathrm{e}^{-H \tau_{0}}\right| x_{i}\right\rangle=N \int[\mathrm{D} x] \mathrm{e}^{-S[x(\tau)]},
$$

where [D $x$ ] denotes integration over all functions $x(\tau)$, obeying the boundary conditions: $x\left(-\tau_{0} / 2\right)=x_{i}, x\left(\tau_{0} / 2\right)=x_{f}$. We can say that, if $\tilde{x}(\tau)$ is any function obeying the boundary conditions, then a general function obeying the bonduary conditions can be written as [1]:

$$
x(\tau)=\tilde{x}(\tau)+\sum_{n} c_{n} x_{n}(\tau)
$$

where $x_{n}$ are a complete set of real, orthonormal functions vanishing at the boundaries:

$$
\begin{gathered}
\int_{-\tau_{0} / 2}^{T_{0} / 2} \mathrm{~d} \tau x_{n}(\tau) x_{m}(\tau)=\delta_{n m}, \\
x_{n}\left( \pm \frac{\tau_{0}}{2}\right)=0 .
\end{gathered}
$$

Then, the measure $[\mathrm{D} x]$ is defined by $[1]$ :

$$
[\mathrm{D} x]=\prod_{n} \frac{\mathrm{d} c_{n}}{\sqrt{2 \pi}} .
$$

The right-hand side of (1.5) is of interest, because it can be evaluted in the semiclassical limit. In this case the functional integral is dominated by the stationary points of $S$, in our case instantons. For simplicity, let us assume that there is only one instanton, which we denote by $\tilde{x}(\tau)$. Then we have:

$$
S[\tilde{x}(\tau)]=S_{0},
$$




$$
N \int[\mathrm{D} x] \mathrm{e}^{-S} \propto \mathrm{e}^{-S_{0}}
$$

and

$$
\delta S=S[\tilde{x}(\tau)+\delta x(\tau)]-S[\tilde{x}(\tau)]=\int_{\tau_{0} / 2}^{\tau_{0} / 2} \mathrm{~d} \tau \delta x(\tau)\left[-\frac{\mathrm{d}^{2} \tilde{x}}{\mathrm{~d} \tau^{2}}+V^{\prime}(\tilde{x})\right]=0,
$$

where

$$
V^{\prime}=\frac{\mathrm{d} V}{\mathrm{~d} x}
$$

Hence

$$
\frac{\mathrm{d}^{2} x}{\mathrm{~d} \tau^{2}}=V^{\prime}(\tilde{x})
$$

This is the equation of motion for a particle of unit mass moving in a potential minus $V$.

Let us choose $x_{n}(1.6)$ to be eigenfunctions of the second variational derivative of $S$ at $\tilde{x}(\tau)$ :

$$
S[\tilde{x}(\tau)+\delta x(\tau)]=S_{0}+\int_{-\tau_{0} / 2}^{\tau_{0} / 2} \mathrm{~d} \tau \delta x(\tau)\left[-\frac{1}{2} \frac{\mathrm{d}^{2}}{\mathrm{~d} \tau^{2}} \delta x(\tau)+\frac{1}{2} V^{\prime \prime}(\tilde{x}) \delta x(\tau)\right]
$$

thus we get

$$
-\frac{\mathrm{d}^{2}}{\mathrm{~d} \tau^{2}} x_{n}(\tau)+V^{\prime \prime}(\tilde{x}) x_{n}(\tau)=\varepsilon_{n} x_{n}(\tau)
$$

and the action (1.12) takes the simple form:

$$
S=S_{0}+\frac{1}{2} \sum_{n} \varepsilon_{n} c_{n}^{2}
$$

finally we obtain:

$$
\left\langle x_{f}\left|\mathrm{e}^{-H \tau_{0}}\right| x_{i}\right\rangle=\mathrm{e}^{-S_{0}} N \prod_{n} \varepsilon_{n}^{-1 / 2}
$$

or written in another way:

$$
\left\langle x_{f}\left|\mathrm{e}^{-H \tau_{0}}\right| x_{i}\right\rangle=\mathrm{e}^{-S_{0}} N\left[\operatorname{det}\left(-\frac{\mathrm{d}^{2}}{\mathrm{~d} \tau^{2}}+V^{\prime \prime}(\tilde{x})\right)\right]^{-1 / 2}
$$

If there are several instantons, in general we have to sum over all of them [4]. Of course we are assuming here that all the eigenvalues are positive, but because of time translation invariance Eq. (1.13) possesses an eigenfunction of eigenvalue zero (we denote them $x_{0}$ and $\varepsilon_{0}$ correspondingly). Thus:

$$
S\left[\tilde{x}\left(\tau, \tau_{\mathrm{c}}\right)\right]-S\left[\tilde{x}\left(\tau, \tau_{\mathrm{c}}+\delta \tau_{\mathrm{c}}\right)\right]=0,
$$

where $\tau_{c}-$ the centre of the instanton. Hence

$$
x_{0}(\tau) \sim \tilde{x}\left(\tau, \tau_{\mathrm{c}}\right)-\tilde{x}\left(\tau, \tau_{\mathrm{c}}+\delta \tau_{\mathrm{c}}\right)
$$


and finally, with the normalization factor from (1.7):

$$
x_{0}(\tau)=S_{0}^{-1 / 2} \frac{\mathrm{d}}{\mathrm{d} \tau} \tilde{x}(\tau) .
$$

It seems that this eigenfunction generates a disaster, because integration over the corresponding expansion coefficient $c_{0}$ in Eq. (1.6) gives an infinity. Fortunately, we can do this integration by means of integrating over the location of the centre of the instanton. The change of $x(\tau)$, induced by a small change in the expansion coefficient $c_{0}$, is:

$$
\mathrm{d} x(\tau)=x_{0}(\tau) \mathrm{d} c_{0} .
$$

On the other hand, the change of $x(\tau)$, induced by a small change in the location of the centre $\tau_{\mathfrak{c}}$, is:

$$
\mathrm{d} x(\tau)=\frac{\mathrm{d} \tilde{x}}{\mathrm{~d} \tau_{\mathrm{c}}} \mathrm{d} \tau_{\mathrm{c}},
$$

hence

$$
\mathrm{d} c_{0}=S_{0}^{1 / 2} \mathrm{~d} \tau_{\mathrm{c}} .
$$

Thus, in evaluating the determinant, we should not include the zero eigenvalue, but we should include the factor $S_{0}^{1 / 2}$.

\section{The triple-well and instantons}

Now we turn to the triple-well of Fig. 1 . There the potential of the triple-well has the exact form:

$$
V(x)=\lambda x^{2}\left(\eta^{2}-x^{2}\right)^{2} .
$$

Using Eq. (1.11) we get the two instantons:

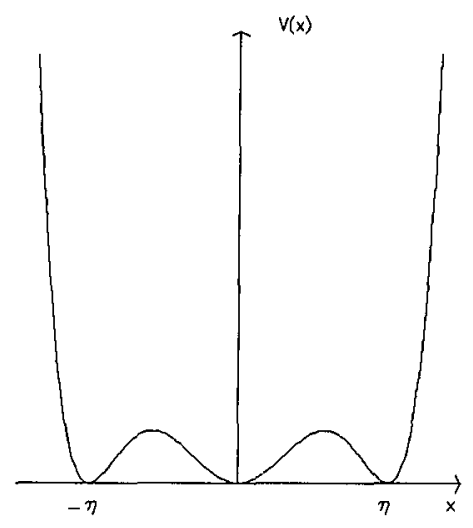

Fig. 1. The potential of the triple-well shape: $V(x)=\lambda x^{2}\left(\eta^{2}-x^{2}\right)^{2}$.

$$
\tilde{x}=\eta\left\{1+\exp \left[2 \omega\left(\tau-\tau_{\mathrm{c}}\right)\right]\right\}^{-1 / 2} \exp \left[\omega\left(\tau-\tau_{\mathrm{c}}\right)\right]
$$


and

$$
\tilde{x}=-\eta\left\{1+\exp \left[-2 \omega\left(\tau-\tau_{\mathrm{c}}\right)\right]\right\}^{-1 / 2} \exp \left[-\omega\left(\tau-\tau_{\mathrm{c}}\right)\right],
$$

where: $\omega^{2}=2 \lambda \eta^{4}$ is the constant, $\tau_{\mathrm{c}}$ is the location of the centre of the instanton.

Because we deal with the potential turned upside down (see Eq. (1.11)), we can say that two such solutions are those in which the particle stays fixed at the top of the hill at $x=0$ (instanton denoted by $\tilde{x}$ for $\tau=-(1 / 2) \tau_{0}$ ) or at the top of the hill at $x=-\eta$ (instanton denoted by $\tilde{x}_{1}$ for $\tau=-(1 / 2) \tau_{0}$ ). However, there is another interesting solution, the one where a particle starts at the top of one hill (say the left one) at time $-\tau_{0} / 2$ and moves to the next hill at time $\tau_{0} / 2$.

We shall focus on only one instanton ( the both instantons cause the same effects which are in our interest.

We start with the action $S_{0}[\tilde{x}]$ :

$$
S_{0}[\tilde{x}]=\int_{-\infty}^{+\infty} \mathrm{d} \tau\left[\frac{1}{2}\left(\frac{\mathrm{d} \tilde{x}}{\mathrm{~d} \tau}\right)^{2}+V(\tilde{x})\right]=\frac{1}{4} \omega \eta^{2}
$$

and next we have to solve Eq. (1.13):

$$
-\frac{\mathrm{d}^{2}}{\mathrm{~d} \tau^{2}} x_{n}(\tau)+\frac{\lambda n^{4}}{2 \cosh ^{2}(\omega \tau)}\left(4 \mathrm{e}^{2 \omega \tau}+\mathrm{e}^{-2 \omega \tau}-10\right) x_{n}(\tau)=\varepsilon_{n} x_{n}(\tau)
$$

with substitutions $x_{n}(\tau)=F_{n}(x), \quad x=\mathrm{e}^{2 \omega \tau}$, we obtain:

$$
F_{n}^{\prime \prime}(x)+\frac{1}{x} F_{n}^{\prime}(x)-C\left[\frac{B}{x^{2}}+\frac{4 x+9}{(x+1)^{2}}-\frac{4}{x}\right] F_{n}(x)=0,
$$

where the prime denotes differentiation with respect to $x$ and

$$
B=\frac{1}{3}\left(1-\frac{\varepsilon_{n}}{\omega^{2}}\right), \quad C=\frac{3}{4} .
$$

Using the transformation $z=x+1$, we arrive at the Fuchsian equation with three singular points:

$$
F_{n}^{\prime \prime}(z)+\frac{1}{z-1} F_{n}^{\prime}(z)-C\left[\frac{B}{(z-1)^{2}}+\frac{4}{z}+\frac{5}{z^{2}}-\frac{4}{z-1}\right] F_{n}(z)=0
$$

Here the prime denotes differentiation with respect to $z$ and the roots of the indicial equation (excluding the root for $z=\infty$ ) are equal to:

$$
\begin{gathered}
\alpha_{1}=5 / 2, \quad \beta_{1}=1 / 2(3 B)^{1 / 2}, \\
\alpha_{2}=-3 / 2, \quad \beta_{2}=-1 / 2(3 B)^{1 / 2} .
\end{gathered}
$$

If we choose

$$
F_{n}(z)=z^{p}(z-1)^{-q} w(z)
$$

where

$$
p=-3 / 2, q=-1 / 2(3 B)^{1 / 2}
$$


then we get:

$$
w^{\prime \prime}+\frac{3+(1-3-2 q) z}{z(z-1)} w^{\prime}+\frac{3 / 2+3 q}{z(z-1)} w=0
$$

and hypergeometric equation has the form:

$$
w^{\prime \prime}+\frac{-\gamma+(1+\alpha+\beta) z}{z(z-1)} w^{\prime}+\frac{\alpha \beta}{z(z-1)} w=0
$$

Thus in our case:

$$
\alpha \beta=3 / 2+3 q, \quad \alpha+\beta=-3-2 q, \quad \gamma=-3 .
$$

This implies that:

$$
\alpha=3 / 2-q+\left(q^{2}+3 / 4\right)^{1 / 2}, \quad \beta=-3 / 2-q-\left(q^{2}+3 / 4\right)^{1 / 2}
$$

and it leads us to the normalizable solutions only if $\alpha=-n$, where $n$ is a positive integer (that is why we have dropped the other values of $\alpha$ and $\beta$ coming from (2.8)). Thus:

$$
q=\frac{3 / 4-(3 / 2-n)^{2}}{2(3 / 2-n)^{2}}
$$

and because $F_{n}(z)$ must be finite for $z=1\left(\tau=-\tau_{0} / 2\right)$, and also for $z=\infty$ $\left(\tau=\tau_{0} / 2\right)$, then we get the two conditions $q<0,-3 / 2-q+n<0$, which have to be satisfied at the same time.

It is true only for $n=0$, or (what is the same) for $\varepsilon=0$, and gives us the eigenfunction [see Eq. (2.4)]:

$$
x_{0}(\tau)=2 \omega^{1 / 2}\left\{1+\exp \left[2 \omega\left(\tau-\tau_{c}\right)\right]\right\}^{-3 / 2} \exp \left[\omega\left(\tau-\tau_{c}\right)\right],
$$

what corresponds with Eq. (1.18).

The second linearly independent solution of the hypergeometric equation is:

$$
w_{2}=z^{1-\gamma} F(\alpha-\gamma+1, \beta-\gamma+1,2-\gamma, z)
$$

and leads to the condition:

$$
q=\frac{(n+5 / 2)^{2}-3 / 4}{2(n+5 / 2)}
$$

where $q>0$ for every $n \geq 0$.

Thus $w_{2}$ does not give normalizable solutions.

Now we turn to Eq. (1.15):

$$
\begin{gathered}
\left\langle x_{f}\left|\mathrm{e}^{-H \tau_{0}}\right| x_{i}\right\rangle=N\left[\operatorname{det}\left(-\frac{\mathrm{d}^{2}}{\mathrm{~d} \tau^{2}}+\omega^{2}\right)\right]^{-1 / 2} \\
\times \frac{\operatorname{det}^{-1 / 2}\left[-\mathrm{d}^{2} / \mathrm{d} \tau^{2}+V^{\prime \prime}(\tilde{x})\right]}{\operatorname{det}^{-1 / 2}\left[-\mathrm{d}^{2} / \mathrm{d} \tau^{2}+\omega^{2}\right]} \mathrm{e}^{-S_{0}}
\end{gathered}
$$


what means normalization to the harmonic oscillator. In accordance with Eq. (1.21) we get:

$$
\frac{\operatorname{det}^{-1 / 2}\left[-\mathrm{d}^{2} / \mathrm{d} \tau^{2}+V^{\prime \prime}(\tilde{x})\right]}{\operatorname{det}^{-1 / 2}\left[-\mathrm{d}^{2} / \mathrm{d} \tau^{2}+\omega^{2}\right]}=\left(\frac{S_{0}}{2 \pi}\right)^{1 / 2} K^{-1 / 2} \omega \mathrm{d} \tau_{\mathrm{c}}
$$

where

$$
K=\frac{\operatorname{det}^{\prime}\left[-\mathrm{d}^{2} / \mathrm{d} \tau^{2}+V^{\prime \prime}(\tilde{x})\right]}{\omega^{-2} \operatorname{det}\left[-\mathrm{d}^{2} / \mathrm{d} \tau^{2}+\omega^{2}\right]}
$$

and $\operatorname{det}^{\prime}$ indicates that the zero eigenvalue is to be omitted when computing the determinant.

As we know, instantons are well-localized objects. It means that, besides one-instanton solutions of the equation of motion, there are also solutions consisting of strings of widely separated instantons. Therefore, we shall evaluate the functional integral by summing over all such configurations, with $n$ instantons centred at different $\tau_{i}$, where:

$$
\frac{\tau_{0}}{2}>\tau_{1}>\tau_{2}>\tau_{3}>\cdots>\tau_{n}>-\frac{\tau_{0}}{2}
$$

and

$$
\begin{array}{r}
N\left[\operatorname{det}\left(\frac{\mathrm{d}^{2}}{\mathrm{~d} \tau^{2}}+\omega^{2}\right)\right]^{-1 / 2}\left(\frac{S_{0}}{2 \pi K}\right)^{1 / 2} \mathrm{e}^{-S_{0}} \omega \mathrm{d} \tau_{c} \longrightarrow \\
N\left[\operatorname{det}\left(-\frac{\mathrm{d}^{2}}{\mathrm{~d} \tau^{2}}+\omega^{2}\right)\right]^{-1 / 2}\left[\left(\frac{S_{0}}{2 \pi K}\right)^{1 / 2} \mathrm{e}^{-S_{0}}\right]^{n} \prod_{i=1}^{n}\left(\omega \mathrm{d} \tau_{i}\right) .
\end{array}
$$

Thus we must integrate over the locations of the centres

$$
\prod_{i=1}^{n}\left(\omega \mathrm{d} \tau_{i}\right) \longrightarrow \int_{-\tau_{0} / 2}^{\tau_{0} / 2} \omega \mathrm{d} \tau_{1} \int_{-\tau_{0} / 2}^{\tau_{1}} \omega \mathrm{d} \tau_{2} \cdots \int_{-\tau_{0} / 2}^{\tau_{n-1}} \omega \mathrm{d} \tau_{n}=\frac{\left(\omega \tau_{0}\right)^{n}}{n !}
$$

It is easy to show that $[3,4]$ :

$$
N\left[\operatorname{det}\left(-\frac{\mathrm{d}^{2}}{\mathrm{~d} \tau^{2}}+\omega^{2}\right)\right]^{-1 / 2}=\left(\frac{\omega}{\pi}\right)^{1 / 2} \exp \left(-\frac{\omega \tau_{0}}{2}\right) .
$$

We arrive at the expression:

$$
\left\langle x_{f}\left|\exp \left(-H \tau_{0}\right)\right| x_{i}\right\rangle=\left(\frac{\omega}{\pi}\right)^{1 / 2} \exp \left(-\frac{\omega \tau_{0}}{2}\right)\left[\left(\frac{S_{0}}{2 \pi K}\right)^{1 / 2} \mathrm{e}^{-S_{0}}\right]^{n} \frac{\left(\omega \tau_{0}\right)^{n}}{n !} .
$$

We will use this equation in the following way: if we start at $-\eta$ and wish to end up back at $-\eta, n$ must be even. Likewise, if we start at $\eta$ and wish to end up at $-\eta, n$ must be odd. Hence

$$
\left\langle-\eta\left|\mathrm{e}^{-H \tau_{0}}\right|-\eta\right\rangle=2 \sum_{n=0,2, \ldots}\left(\frac{\omega}{\pi}\right)^{1 / 2} \exp \left(-\frac{\omega \tau_{0}}{2}\right) \frac{\left(\mathrm{d} \omega \tau_{0}\right)^{n}}{n !}=
$$




$$
\begin{gathered}
=2\left(\frac{\omega}{\pi}\right)^{1 / 2} \exp \left(-\frac{\omega \tau_{0}}{2}\right) \cosh \left(\omega \tau_{0} d\right) \\
\left\langle-\eta\left|\mathrm{e}^{-H \tau_{0}}\right| \eta\right\rangle=2 \sum_{n=1,3, \ldots}\left(\frac{\omega}{\pi}\right)^{1 / 2} \exp \left(-\frac{\omega \tau_{0}}{2}\right) \frac{\left(\mathrm{d} \omega \tau_{0}\right)^{n}}{n !}= \\
=2\left(\frac{\omega}{\pi}\right)^{1 / 2} \exp \left(-\frac{\omega \tau_{0}}{2}\right) \sinh \left(\omega \tau_{0} d\right)
\end{gathered}
$$

where

$$
d=\left(\frac{S_{0}}{2 \pi K}\right)^{1 / 2} \mathrm{e}^{-S_{0}}
$$

or

$$
\left\langle-\eta\left|\mathrm{e}^{-H \tau_{0}}\right| \pm \eta\right\rangle=\left(\frac{\omega}{\pi}\right)^{1 / 2} \exp \left(-\frac{\omega \tau_{0}}{2}\right)\left[\exp \left(\omega \tau_{0} d\right) \pm \exp \left(-\omega \tau_{0} d\right)\right] .
$$

comparing this to Eq. (1.3), we see that we have two low-lying energy eigenstates, with energies:

$$
E_{ \pm}=\frac{\omega}{2} \pm \frac{\eta \omega^{3 / 2}}{2}(2 \pi K)^{-1 / 2} \exp \left[-\frac{\omega \eta^{2}}{2}\right]
$$

It is time to evalute the primed determinant, one with the zero eigenvalue omitted [4].

Now, let $W^{\langle 1\rangle}$ and $W^{\langle 2\rangle}$ be two functions of $\tau$ and let $\Psi_{\varepsilon}^{\langle 1,2\rangle}$ be the associated solutions of equation given below:

$$
\left[-\frac{\mathrm{d}^{2}}{\mathrm{~d} \tau^{2}}+W\right] \Psi=\varepsilon \Psi
$$

with the boundary conditions:

$$
\Psi\left[-\frac{\tau_{0}}{2}\right]=0, \quad \frac{\mathrm{d}}{\mathrm{d} \tau} \Psi\left[-\frac{\tau_{0}}{2}\right]=1 .
$$

Then it is proved that [4]:

$$
\operatorname{det}\left[\frac{-\mathrm{d}^{2} / \mathrm{d} \tau^{2}+W^{(1)}-\varepsilon}{-\mathrm{d}^{2} / \mathrm{d} \tau^{2}+W^{(2)}-\varepsilon}\right]=\frac{\Psi_{\varepsilon}^{\langle 1\rangle}\left(\tau_{0} / 2\right)}{\Psi_{\varepsilon}^{(2)}\left(\tau_{0} / 2\right)} .
$$

We will do our task by evaluating the full determinant within a finite interval, $\left[-\tau_{0} / 2, \tau_{0} / 2\right]$, dividing it by its smallest eigenvalue, $\varepsilon_{0}$, and then letting $\tau_{0}$ go to infinity. Thus we must construct solutions of:

$$
\left[-\frac{\mathrm{d}^{2}}{\mathrm{~d} \tau^{2}}+V^{\prime \prime}(\tilde{x})\right] \Psi_{\varepsilon}=\varepsilon \Psi_{\varepsilon} .
$$

We already know one solution with $\varepsilon=0$,

$$
x_{0}=\left\{\begin{array}{lll}
A \mathrm{e}^{-\omega|\tau|} & \text { for } & \tau \rightarrow-\infty \\
A \mathrm{e}^{-2 \omega|\tau|} & \text { for } & \tau \rightarrow+\infty
\end{array}\right.
$$


where $A=2 \omega^{1 / 2}$.

A second solution with $\varepsilon=0$ we denote by $y_{0}$. It will be convenient to normalize $y_{0}$ such that its Wronskian with $x_{0}$ is given by:

$$
x_{0} \partial_{\tau} y_{0}-y_{0} \partial_{\tau} x_{0}=2 A^{2} \text {, }
$$

thus

$$
y_{0}=\left\{\begin{array}{lll}
-(A / \omega) \mathrm{e}^{-\omega|\tau|} & \text { for } & \tau \rightarrow-\infty \\
-(A / 2 \omega) \mathrm{e}^{-2 \omega|\tau|} & \text { for } & \tau \rightarrow+\infty
\end{array}\right.
$$

Now we can construct the function $\Psi_{0}$ for large $\tau_{0}$ :

$$
y_{0}(\tau)=(2 A \omega)^{-1}\left(x_{0}+\omega \mathrm{e}^{-\omega \tau_{0}} y_{0}\right)
$$

and

$$
\Psi_{0}\left[\frac{\tau_{0}}{2}\right]=\frac{1}{4 \omega} .
$$

To find the lowest eigenvalue, we find $\Psi_{\varepsilon}(\tau)$ for small $\varepsilon$. This can be done by turning Eq. (2.17) into an integral equation and iterating [4]:

$$
\Psi_{\varepsilon}(\tau)=\Psi_{0}(\tau)-\left(2 A^{2}\right)^{-1} \varepsilon \int_{-\tau / 2}^{\tau_{0} / 2} \mathrm{~d} \tau^{\prime}\left[y_{0}(\tau) x_{0}\left(\tau^{\prime}\right)-x_{0}(\tau) y_{0}\left(\tau^{\prime}\right)\right] \Psi_{0}\left(\tau^{\prime}\right)
$$

and

$$
\Psi_{\varepsilon}\left(\tau_{0} / 2\right)=\Psi_{0}\left(\tau_{0} / 2\right)-\left(2 A^{2}\right)^{-1} \varepsilon I
$$

where

$$
I=\int_{-\tau_{0} / 2}^{\tau_{0} / 2} \mathrm{~d} \tau\left[y_{0}\left(\tau_{0} / 2\right) x_{0}(\tau)-x_{0}\left(\tau_{0} / 2\right) y_{0}(\tau)\right] \Psi_{0}(\tau) .
$$

For large $\tau_{0}$ is $I=\left(1 / 4 \omega^{2}\right) \mathrm{e}^{\omega \tau_{0}}$. Thus we get:

$$
\varepsilon_{0}=2 A^{2} I^{-1} \Psi_{0}\left(\tau_{0} / 2\right)
$$

and

$$
K=\frac{\dot{\Psi}_{0}\left(\tau_{0} / 2\right)}{\varepsilon_{0} \Psi_{0}^{h}\left(\tau_{0} / 2\right)} \omega^{2}
$$

where [4]:

$$
\Psi_{0}^{h}\left(\tau_{0} / 2\right)=\omega^{-1} \sinh \left(\omega \tau_{0}\right) \approx \omega^{-1} \frac{\mathrm{e}^{\omega \tau_{0}}}{2}
$$

Finally

$$
K=\frac{\omega}{4 A^{2}}=\frac{1}{16}
$$

and the energy eigenvalues are given by:

$$
E_{ \pm}=\frac{\omega}{2} \pm 4 \omega\left(\frac{S_{0}}{2 \pi}\right)^{1 / 2} \exp \left(-\frac{\omega^{2}}{4(2 \lambda)^{1 / 2}}\right)
$$

What about the second instanton solution denoted by $\tilde{x}_{1}$ ? It is sufficient to show that it produces the same action $S_{0}$ and the same primed determinant as $\tilde{x}$. We 
only notice equivalence of the action for the both instantons. As to computing the primed determinant we proceed the same way as before.

From Eq. (1.18) we get:

$$
x_{01}=\left\{\begin{array}{lll}
A \mathrm{e}^{-\omega|\tau|} & \text { for } & \tau \rightarrow+\infty \\
A \mathrm{e}^{-2 \omega|\tau|} & \text { for } & \tau \rightarrow-\infty
\end{array}\right.
$$

where $A=2 \omega^{1 / 2}$.

The second linearly independent solution of Eq. (2.17) has the form:

$$
y_{01}=\left\{\begin{array}{lll}
(A / \omega) \mathrm{e}^{\omega|\tau|} & \text { for } & \tau \rightarrow+\infty \\
(-A / 2 \omega) \mathrm{e}^{2 \omega|\tau|} & \text { for } & \tau \rightarrow-\infty
\end{array}\right.
$$

and the function analogous to Eq. (2.19) is:

$$
\Psi_{0}(\tau)=(4 \omega A)^{-1}\left(\mathrm{e}^{\omega \tau_{0} / 2} x_{01}(\tau)+2 \omega \mathrm{e}^{-3 \omega \tau_{0} / 2} y_{01}(\tau)\right)
$$

For large $\tau_{0}$

$$
\Psi_{0}\left[\frac{\tau_{0}}{2}\right]=\frac{1}{4 \omega}
$$

and finally $K_{1}=1 / 16$.

Now we can draw the conclusion that the both instantons cause the same effects which are of interest to us, what means that Eqs. (2.13) are valid.

\section{The triple-well and variational methods}

Here we are dealing with a verage energy of the form:

$$
\varepsilon=\int \phi^{*}(x) H \phi(x) \mathrm{d} x
$$

with normalization

$$
\int \phi^{*}(x) \phi(x) \mathrm{d} x=1
$$

By means of expansion

$$
\phi=\sum_{k} a_{k} \Psi_{k},
$$

we can minimize an energy with respect to $a_{k}$. We choose

$$
\phi=a_{-1} \exp \left[-\omega(x+\eta)^{2}\right]+a_{0} \exp \left(-\omega x^{2}\right)+a_{1} \exp \left[-\omega(x-\eta)^{2}\right]
$$

with Hamiltonian equal to

$$
H=-\frac{1}{2} \frac{\mathrm{d}^{2}}{\mathrm{~d} x^{2}}+\lambda x^{2}\left(\eta^{2}-x^{2}\right)^{2} .
$$


Hence we get the conditions:

$$
a_{0}=0, \quad a_{1}=a_{-1}, \quad a_{1}^{2}=\left(\frac{2 \pi}{\omega}\right)^{-1 / 2}\left[1+\exp \left(-2 \omega \eta^{2}\right)\right]
$$

or

$$
a_{0}=0, \quad a_{1}=-a_{-1}, \quad a_{1}^{2}=\left(\frac{2 \pi}{\omega}\right)^{-1 / 2}\left[1-\exp \left(-2 \omega \eta^{2}\right)\right],
$$

and both of them minimize (3.1).

Finally, we arrive at two low-lying energies:

$$
\varepsilon_{ \pm}=\frac{\omega}{2} \pm 6 \omega S_{0} \exp \left(-\frac{2 \omega^{2}}{(2 \lambda)^{1 / 2}}\right)
$$

Comparing this to (2.26) we see, that both expressions are very similar to each other, and that we are in a position to equalize them by means of a properly selected test function in the variational method.

\section{Discussion}

We have described, in the context of particle mechanics, the new method, where the instantons play a central role. At the first sight this method seems to be more awkward than the standard methods of one-dimensional quantum mechanics. However, it has a great advantage of being immediately generalizable to quantum field theory.

We would also like to know where are the limits of applicability of instantons as the method of one-dimensional quantum mechanics. We can solve this problem considering the potential shown in Fig. 2. Unfortunately, in this case we have to

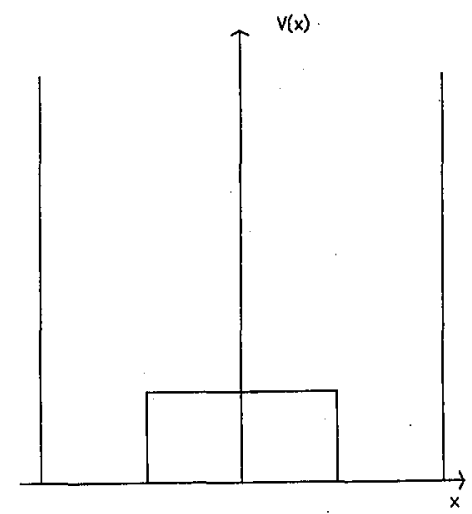

Fig. 2. An example of the potential for which the instanton method is not useful. 
work out the equation:

$$
\frac{\mathrm{d}^{2} F(\tau)}{\mathrm{d} \tau^{2}}+V_{0}\left[\delta^{\prime}(k \tau+1)-\delta^{\prime}(k \tau-1)\right] F(\tau)=\varepsilon F(\tau),
$$

where $V_{0}, k, l$ are constans, and the prime denotes differentiation with respect to $\tau$.

This can be done only by estimation of $\delta$. Of course it makes the potential smooth and changes the whole problem. Thus, we can draw the conclusion, that the instanton method is not adequate to solve problems with sharp-edged potentials.

\section{Acknowledgments}

The author is highly indebted to Professor H. Arodź of the Jagellonian University, who has contributed throughout the course of this work.

\section{References}

[1] R. Feynman, A. Hibbs, Quantum Mechanics and Path Integrals, Mc Graw-Hill, New York 1965.

[2] A. Belavin, A. Polyakov, A. Schwartz, Y. Tyupkin, Phys. Lett. B 59, 85 (1975).

[3] A. Vainshtain, W. Zacharov, W. Novikov, M. Shifman, Usp. Fiz. Nauk 136, 553 (1982).

[4] S. Coleman, in: Aspects of Symmetry, Univ. Press, Cambrige 1985.

[5] R. Rajaraman, Solitons and Instantons, North-Hollan,d Amsterdam 1982. 